\title{
Semantic associations between arithmetic and space: Evidence from temporal order judgements
}

\author{
Michael Andres $^{1,2} \cdot$ Samuel Salvaggio ${ }^{1,2} \cdot$ Nathalie Lefèvre $^{1,3} \cdot$ Mauro Pesenti $^{1,2} \cdot$ Nicolas Masson $^{1,2,4}$ (DD
}

Published online: 16 September 2019

(C) The Psychonomic Society, Inc. 2019

\begin{abstract}
Spatial biases associated with subtraction or addition problem solving are generally considered as reflecting leftward or rightward attention shifts along a mental numerical continuum, but an alternative hypothesis not implying spatial attention proposes that the operator (plus or minus sign) may favour a response to one side of space (left or right) because of semantic associations. We tested these two accounts in a series of temporal order judgement experiments that consisted in the auditory presentation of addition or subtraction problems followed $200 \mathrm{~ms}$ (Experiments 1-2) or $800 \mathrm{~ms}$ (Experiment 3) later by the display of two lateralized targets in close temporal succession. To dissociate the side where the operation first brought their attention from the side they had to respond to, we asked participants to report which of the left or right target appeared first or last on screen. Under the attentionorienting account, addition should elicit more rightward responses than subtraction when participants have to focus on the first target, but more leftward responses when they have to focus on the last target, because the latter is opposite to the side where the operation first brought their attention. Under the semantic account, addition should elicit more rightward responses than subtraction, no matter the focus is on the first or last target, because participants should systematically favour the side conceptually linked to the operator. The results of the three experiments converge to indicate that, in lateralized target detection tasks, the spatial biases induced by arithmetic operations stem from semantic associations.
\end{abstract}

Keywords mathematical cognition $\cdot$ semantic priming $\cdot$ spatial cognition $\cdot$ attention $\cdot$ number processing

Stimulus-response compatibility effects show that numbers are coded in spatial coordinates, with associations between small numbers and the left side of space and large numbers and the right side of space (Dehaene, 1992). These associations can be flexibly adapted in short-term memory (van Dijck \& Fias, 2011) - for example, as a function of numerical range (Fias, Brysbaert, Geypens \& d'Ydewalle, 1996) or of the visuospatial medium participants refer to (Bächtold, Baumüller,

Nicolas Masson

nicolas.masson@uclouvain.be

1 Psychological Sciences Research Institute, Université catholique de Louvain, Place Cardinal Mercier, 10,

B-1348 Louvain-la-Neuve, Belgium

2 Institute of Neuroscience, Université catholique de Louvain, Louvain-la-Neuve, Belgium

3 Louvain Institute of Data Analysis and Modeling in economics and statistics, Université catholique de Louvain,

Louvain-la-Neuve, Belgium

4 Department of Psychology, University of Tübingen, Tübingen, Germany
\& Brugger, 1998). This raises the question of how numbers are manipulated to perform a given task. Mental arithmetic provides a paradigmatic case to study this question as many adults rely on visuospatial strategies to face the cognitive load of calculation (Hayes, 1973). Brain imaging studies also show that the activations induced by complex calculation overlap those observed during visuospatial short-term memory and mental visual imagery tasks (Zago et al., 2001; Zago et al., 2008) or horizontal saccades (Knops, Thirion, Hubbard, Michel, \& Dehaene, 2009b). Chronometric studies further show that solving subtraction and addition problems facilitates the detection of left and right targets, respectively (Liu, Cai, Verguts, \& Chen, 2017b; Liu, Verguts, Li, Ling, \& Chen, 2017a; Masson \& Pesenti, 2014) or influences the trajectory of hand movements (Marghetis, Núñez, \& Bergen, 2014; Pinheiro-Chagas, Dotan, Piazza, \& Dehaene, 2017), while the latency of arithmetic problem solving is congruently influenced by flickering lateralized targets (Masson \& Pesenti, 2016), lateralized operand displays (Mathieu, Gourjon, Couderc, Thevenot, \& Prado, 2016), lateralized arm movements (Wiemers, Lindemann, \& Bekkering, 2014), or lateral optokinetic stimulation (Masson, Pesenti, \& Dormal, 2017b). 
These interactions are explained by two types of accounts. Attentional accounts propose that subtraction and addition move attention in opposite directions on a spatial continuum where numbers are aligned in ascending order (Hubbard, Piazza, Pinel, \& Dehaene, 2005; Knops, Thirion, et al., 2009b; McCrink, Dehaene, \& Dehaene-Lambertz, 2007). Semantic accounts do not imply a functional role for spatial attention in the solving procedure, but rather suggest that the operator favours a response to one side of space because plus or minus signs would be conceptually linked to one side of space (e.g., Hartmann, Mast, \& Fischer, 2015; Pinhas \& Fischer, 2008; Pinhas, Shaki, \& Fischer, 2014). The polarity correspondence principle, for example, assigns a positive linguistic polarity to the concepts of right and addition, and a negative linguistic polarity to the concepts of left and subtraction (Gevers, Lammertyn, Notebaert, Verguts, \& Fias, 2006; Gevers, et al., 2010; Proctor \& Cho, 2006).

Attention and semantic accounts are difficult to disentangle in studies in which stimulus display and response mapping are confounded because both accounts lead to the same predictions. Hence, a target displayed on the left (right) side of a screen might be detected faster after a subtraction (addition) either because of an attentional process or a semantic association. In the present study, we addressed this issue by combining mental arithmetic with a temporal order judgement (TOJ) task (Casarotti, Michielin, Zorzi, \& Umiltà, 2007; Stelmach \& Herdman, 1991). Experiment 1 consisted in the auditory presentation of addition or subtraction problems, followed $200 \mathrm{~ms}$ later by the display of left and right targets with no or short temporal asynchronies. Participants had to solve the arithmetic problem and then report which of the left or right target appeared first on the screen. Attention and semantic accounts both predict a larger proportion of right responses when the TOJ follows addition rather than subtraction problems. Experiment 2 used the same procedure, but participants had to report the last target appearing on the screen. Reversing the instruction implies that if spatial biases reflect attention shifts, the results should be opposite to those of Experiment 1 because the participants would identify the last target as the one appearing opposite to the side where the operation first brought their attention, and therefore press the opposite response key. Conversely, if spatial biases reflect an association of concepts in semantic memory, the results should be the same as in Experiment 1 because participants would answer "right" after addition and "left" after subtraction, independently of their attentional focus. In Experiment 3, the left and right targets were displayed $800 \mathrm{~ms}$ after problem offset, and participants were asked to judge the temporal order before they responded to the arithmetic problem in order to maximize the probability they were calculating at the time they made the judgement. In this control experiment, the instruction relative to order discrimination (which target appeared first vs. last?) was manipulated within subject.

\section{Experiment 1}

\section{Method}

Participants Twenty-four right-handed French-speaking participants took part in this experiment (age range: 18-30 years; 20 females). They reported no antecedent of mathematical learning difficulties and had normal or corrected-to-normal vision. The procedures were in accordance with the ethical standards established by the Declaration of Helsinki.

Task and stimuli At each trial, participants had to perform two concurrent tasks: (i) solve an arithmetic problem, and (ii) indicate on which side of the screen (i.e., left or right) the first of two (a)synchronous stimuli appeared. In the arithmetic task, the participants were asked to respond aloud to 2 -digit \pm 1 digit problems presented auditorily through headphones. The list of problems was made of 144 addition and 144 subtraction problems created on the basis of the following considerations: The first operand (O1) ranged between 11 and 79 (addition: 11-69; subtraction: 21-79); within each decade, we selected all numbers with units of $1,2,3,7,8$ or 9 . The second operand $(\mathrm{O} 2)$ was either 4 or 6 . Each decade-unit combination (O1) was associated once with \pm 4 and once with $\pm 6(\mathrm{O} 2)$. Addition and subtraction problems were thus matched for the magnitude of their answer (addition: $45 \pm 18$; subtraction: $45 \pm 18$ ), and each operation involved a carry or borrow procedure in half of the trials. The audio recording of the arithmetic problems was performed by a male voice. The speaker was trained to pronounce each problem with the same pace. Audacity ${ }^{\circledR}$ software was used to mark precisely the onset and offset of each problem on the recording, and the sampled period was then compressed in a WAV file whose duration was set to $1,500 \mathrm{~ms}$. The compression proceeded by increasing the rate of enunciation or by cutting in the silent periods separating the numbers so that the quality of the recording was not altered. When presented to the participants, the duration of the audio recording was thus constant, and the offset of the problem coincided with the end of the recording in all trials. The visual targets were green squares of $0.5^{\circ}$ of visual angle displayed with a stimulus-onset asynchrony (SOA) of $-200,-100,-50$, $-33,-17,0,17,33,50,100$, or $200 \mathrm{~ms}$, where negative values indicate that the left target appeared before the right target. The first target, being the left or right one, always appeared $200 \mathrm{~ms}$ later than the offset of the problem. The participants had to indicate which of the two targets appeared first on the screen by pressing with the index and middle finger of their dominant hand on the left or right arrow keys on a standard keyboard. We collected 12 responses for each combination of SOA (from -200 to $200 \mathrm{~ms}$ ) and operation (addition vs. subtraction). The decade of the first operand, the unit of the second operand, and the carry/borrow procedures were equally counterbalanced over the different conditions of the TOJ task. 
Apparatus The experiment was conducted on a Dell PC equipped with a 17 -inch LCD screen and a refresh rate of 60 Hz. The audio recording of the arithmetic problems was presented through headphones (Sennheiser PC8 USB) equipped with a microphone to record the participant's answer to the problem. Stimulus presentation and response recording were monitored using PsychoPy (Peirce, 2007).

Procedure Participants were seated $60 \mathrm{~cm}$ from the computer screen, with the midline of their face aligned with the centre of the screen and their head positioned in a chin rest to limit movements. The sequence of events was as follows (see Fig. 1). A $0.5-\mathrm{cm}$ height orange fixation square (i.e., $0.5^{\circ}$ ) was presented for $500 \mathrm{~ms}$ in the centre of the screen, followed by the auditory presentation of the arithmetic problem lasting $1,500 \mathrm{~ms}$. Then, $200 \mathrm{~ms}$ after the offset of the audio file, the fixation square disappeared, and the first of the two lateral green squares (i.e., the first target) was flashed for $33 \mathrm{~ms}$ at a $2.5^{\circ}$ eccentricity on one side of the screen; the second target appeared on the opposite side of the screen with an SOA ranging from 0 to $200 \mathrm{~ms}$. Participants were asked to solve the problem as fast as possible and then to respond to the TOJ with a deadline of $6,000 \mathrm{~ms}$ after problem offset. Responses to the arithmetic problems were registered online by the experimenter, and latencies were measured with a voice key from the offset of the auditory file. Responses to the TOJ were recorded on a standard keyboard on which participants had to press left or right keys. The session was made of one training block of 12 trials and six blocks of 48 items. A session lasted approximately 45 minutes.

Data analysis The analyses were performed on the trials where a correct arithmetic answer was given in the prescribed delay.
Four participants were excluded because more than $20 \%$ of their data were discarded based on this criterion. A generalized linear mixed model (GLMM) was used to model the error rates (ER), and a linear mixed model to model response latencies (RL) computed from problem offset, with operation as fixed effect and the differences between participants as a random intercept and the combination of participants and operation as a random slope. A GLMM was used to model the probability of responding "right first" in the remaining participants $(N=20)$ with SOA (logistic function), OPERATION, and their interaction as fixed effects, and the differences between participants as a random intercept and the combination of participants and operation as a random slope. The SOA was expressed as a negative value (from -200 to $-17 \mathrm{~ms}$ ) when the first target was on the left, as a positive value (from 17 to 200 $\mathrm{ms}$ ) when the first target was on the right, and set at zero when the targets were simultaneous. The points of maximal uncertainty (PMU), corresponding to the estimated SOA where participants gave an equal proportion of rightward and leftward responses, were obtained from the intercept (B0) and the slope (B1) revealed by the GLMM using the formula $-\frac{B 0 * \text { operation }}{B 1}$.

\section{Results}

In the arithmetic task, participants were slightly slower and made more errors when they solved subtraction compared to addition problems - mean $\mathrm{RL} \pm S D=2,055 \pm 124$ vs. $1,956 \pm$ $124 \mathrm{~ms}, F(1,5434)=17.161, p<.001 ;$ mean $\mathrm{ER} \pm S D=5 \% \pm$ $1 \%$ vs. $3 \% \pm 1 \%, F(1,5758)=7.957, p=.005$.

In the TOJ task, the GLMM analysis showed a significant effect of OPERATION, $F(1,5432)=13.527, p<.001$, indicating that the right target was more often reported as the first appearing on the screen in addition than in subtraction trials

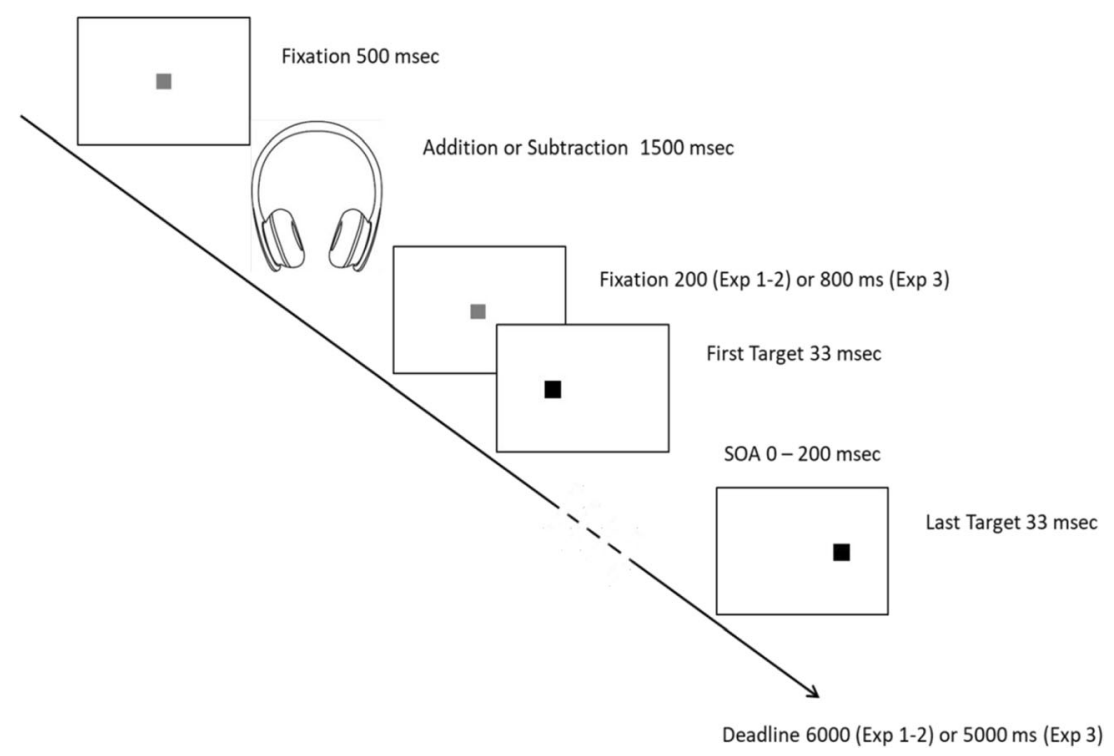

Fig. 1 Time course of one trial in the dual task combining mental arithmetic and temporal order judgement 
(see Fig. 2), and an effect of SOA, $F(1,5432)=896.32, p<$ .001 , indicating that the probability of reporting the right target as the first one increased from $3 \%$ at SOA $-200 \mathrm{~ms}$ (i.e., left before right) to $96 \%$ at SOA $+200 \mathrm{~ms}$ (i.e., right before left). A significant interaction between SOA and OPERATION, $F(1,5432)=5.712, p=.017$, indicated that the increase of right responses with SOA was steeper in the addition than in the subtraction condition, meaning that temporal order was better discriminated when the targets followed addition problems (see Fig. 2). The ER and RL analyses showed that subtraction required more attentional resources than addition, which impacted on discrimination accuracy in temporal order judgements. Further comparisons ( $\alpha=.05$ corrected for multiple SOAs) showed that the probability of reporting the right target was significantly higher after addition than subtraction for SOAs ranging from $-17 \mathrm{~ms}$ to $+200 \mathrm{~ms}$ (see Table 1 ). The PMU was $+1 \mathrm{~ms}$ for addition and $+19 \mathrm{~ms}$ for subtraction.

\section{Experiment 2}

\section{Method}

Participants Twenty-five right-handed French-speaking participants (age range: 18-30 years; 18 females) who had not participated in Experiment 1, had no antecedents of mathematical learning difficulties, and had normal or corrected-tonormal vision participated in this experiment. The procedures were in accordance with the ethical standards established by the Declaration of Helsinki.

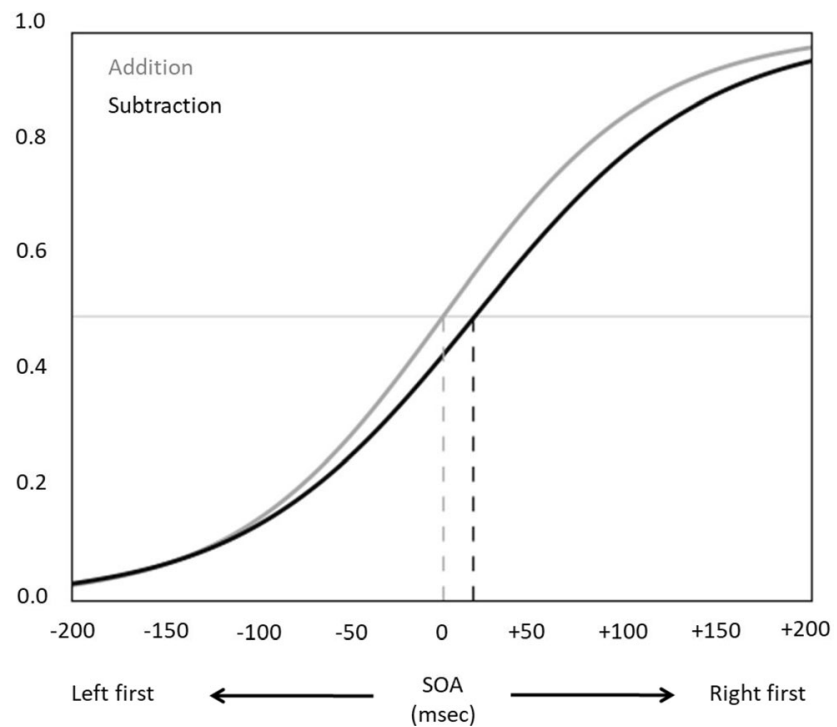

Fig. 2 Probability of reporting the right target as the first, in Experiment 1 , as a function of operation (addition vs. subtraction) and SOA between the left and right targets (from -200 to +200 ). Negative and positive SOAs refer to left-side and right-side precedence, respectively. Dashed lines indicate the PMU for addition (gray) and subtraction (black)
Task, stimuli, apparatus, and procedure Task, stimuli, apparatus, and procedure were the same as in Experiment 1, except that in the TOJ task, participants had to report the side of the last instead of the first target appearing on the screen.

Data analysis The analyses were performed on the trials where a correct arithmetic answer was given in the prescribed delay. Four participants were excluded because more than $20 \%$ of their data were discarded based on this criterion. An additional participant was excluded from the study because he could not concentrate on the task, and another because he misunderstood the instructions of the TOJ and reported the first instead of the last target. A GLMM was used to model the probability of responding "right last" in the remaining participants $(N=$ 19) with SOA (logistic function) OPERATION and their interaction as fixed effects, and the differences between participants as a random intercept and the combination of participants and operation as a random slope.

\section{Results}

In the arithmetic task, participants were slower and made more errors when they solved subtraction compared with addition problems - mean $\mathrm{RL} \pm S D=2,046 \pm 166$ vs. $1,932 \pm 166 \mathrm{~ms}$; $F(1,5002)=23.796, p<.001 ;$ mean $\mathrm{ER} \pm S D=7 \% \pm 1 \%$ vs. $4 \% \pm 1 \%, F(1,5470)=10.511, p=.001)$.

In the TOJ task, the GLMM analysis revealed no significant main effect of OPERATION, $F(1,5000)=2.186, p=.139$. The effect of SOA, $F(1,5000)=855.414, p<.001$, was significant and indicated that the probability of reporting the right target as the last decreased from $98 \%$ at SOA $-200 \mathrm{~ms}$ (i.e., left before right) to $3 \%$ at SOA $+200 \mathrm{~ms}$ (i.e., right before left). The interaction between SOA and OPERATION was also significant, $F(1,5000)=11.641, p=.001$, indicating that the decrease of right responses with SOA was steeper in the addition than in the subtraction condition, meaning that temporal order was better discriminated when the targets followed addition problems (see Fig. 3). Post hoc comparisons showed that the probability of reporting the right target as the last was significantly higher after addition than subtraction for SOAs ranging from $-200 \mathrm{~ms}$ to $-50 \mathrm{~ms}$ (see Table 2). The PMU was $+25 \mathrm{~ms}$ for addition and $+12 \mathrm{~ms}$ for subtraction.

\section{Experiment 3}

The results of Experiments 1 and 2 support a semantic account of the spatial biases induced by arithmetic operations in lateralized detection tasks because addition was found to increase the proportion of rightward responses relative to subtraction, regardless of the instruction about which of the first or last target should be reported. However, because participants were asked to solve the arithmetic problem before 
Table 1 Probability of right-first responses in Experiment 1

\begin{tabular}{|c|c|c|c|c|c|c|c|c|c|c|c|c|}
\hline \multirow[t]{2}{*}{ Operation } & \multicolumn{12}{|c|}{ SOA (ms) } \\
\hline & -200 & -100 & -50 & -33 & -17 & 0 & 17 & 33 & 50 & 100 & 200 & Total \\
\hline Addition & .02 & .14 & .29 & .35 & .42 & .49 & .57 & .64 & .71 & .86 & .97 & .50 \\
\hline Subtraction & .03 & .14 & .26 & .31 & .37 & .43 & .19 & .56 & .62 & .78 & .94 & .45 \\
\hline
\end{tabular}

Note. Boldface values indicate significant differences

responding to the targets, we cannot exclude that they postponed the temporal order judgement and relied on memory processes that would be more vulnerable to semantic influences. The slopes further indicate that temporal order was better discriminated after addition than after subtraction, suggesting that participants might have more resources to focus on the targets after they had solved an addition problem. In order to address these issues, we conducted an additional experiment where temporal order was judged before giving the response to the arithmetic problem and the delay between problem offset and target(s) onset was set to $800 \mathrm{~ms}$ to maximize the probability that participants were calculating at the time of target onset. This delay was chosen to cover the time window where previous studies reported spatial biases (Fischer, Castel, Dodd, \& Pratt, 2003), and the response deadline was reduced to $5 \mathrm{~s}$ (against $6 \mathrm{~s}$ in Experiments 1-2) to encourage participants to answer as fast as possible. Finally, the instruction relative to order discrimination (i.e., Which target comes first $v$ s. last?) was changed across blocks of trials to allow withinsubjects comparisons.

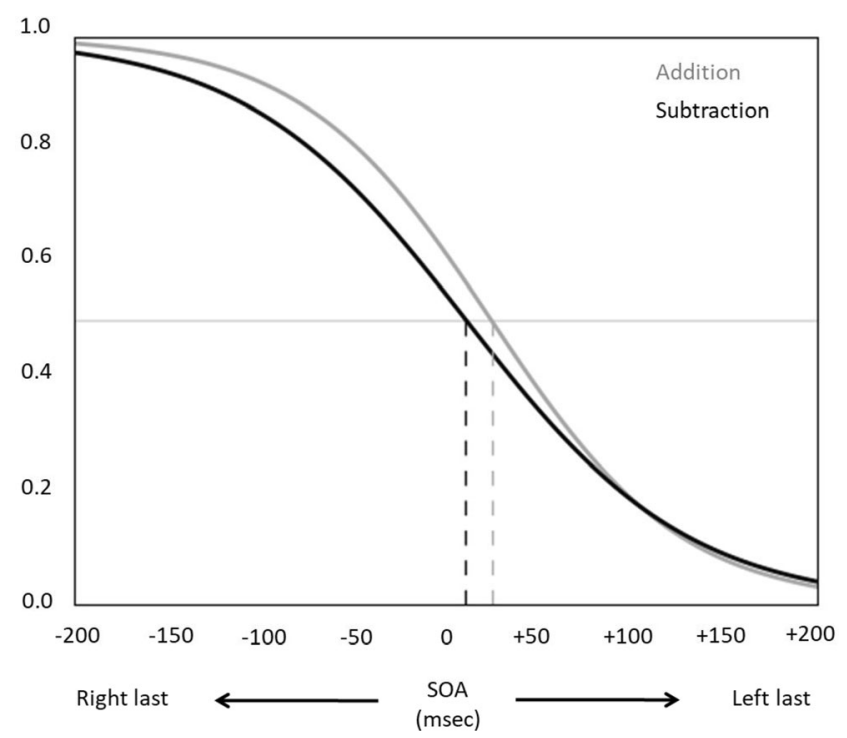

Fig. 3 Probability of reporting the right target as the last, in Experiment 2, as a function of operation (addition vs. subtraction) and SOA between the left and right targets (from -200 to +200 ). Negative and positive SOAs refer to left-side and right-side precedence, respectively. Dashed lines indicate the PMU for addition (gray) and subtraction (black)

\section{Method}

Participants Forty-three right-handed French-speaking participants (age range: 19-31 years; 24 females) took part in this experiment. They did not participate to the previous experiments, reported no antecedents of mathematical learning difficulties and had normal or corrected-to-normal vision. The procedures were in accordance with the ethical standards established by the Declaration of Helsinki.

Task, stimuli, apparatus, and procedure Task, stimuli, apparatus, and procedure were the same as in previous experiments, except that participants were asked to report the first or last target as a function of instruction before giving the answer of the arithmetic problem. Moreover, the delay between the offset of the problem and the onset of the first target was set to $800 \mathrm{~ms}$ to ensure that participants were calculating when the targets appeared on the screen. Finally, the effect of the instruction was tested in a within-subjects design. Participants had to report the first target in three consecutive blocks and the last target in three other blocks. The order of presentation of the two instructions was counterbalanced across participants. The session was thus divided in two parts, each including one training block of 12 trials and three blocks of 48 items. The number of observations per condition was twice as small as in the previous experiments, but the number of participants was twice as large as in previous experiments. Participants were encouraged to perform the tasks as fast as possible with a deadline of $5 \mathrm{~s}$ after problem offset.

Data analysis The analyses were performed on the trials where a correct arithmetic answer was given in the prescribed delay. Three participants were excluded because more than $20 \%$ of their data were discarded based on this criterion, and one participant was excluded because he reported the first target in all trials even when the instruction required him to report the last target. Two other participants did not finish the experiment because they could not concentrate on the task. A GLMM was used to model the probability of reporting the "right first" in the remaining participants $(N=39)$, with SOA (logistic function), OPERATION (addition vs. subtraction), INSTRUCTION (identify the first vs. last target), their interactions as fixed effects, the differences between participants as a random intercept, and the combination of participants and operation as a random 
Table 2 Probability of right-last responses in Experiment 2

\begin{tabular}{|c|c|c|c|c|c|c|c|c|c|c|c|c|}
\hline \multirow[t]{2}{*}{ Operation } & \multicolumn{12}{|c|}{ SOA (ms) } \\
\hline & -200 & -100 & -50 & -33 & -17 & 0 & 17 & 33 & 50 & 100 & 200 & Total \\
\hline Addition & .99 & .93 & .81 & .76 & .69 & .61 & .53 & .46 & .38 & .19 & .03 & .58 \\
\hline Subtraction & .97 & .86 & .73 & .66 & .61 & .54 & .48 & .42 & .36 & .20 & .05 & .53 \\
\hline
\end{tabular}

Note. Boldface values indicate significant differences

slope. For the sake of comparison, all responses were coded as a function of which target was judged as the first, even when the task required participants to focus on the last. Hence, in trials where participants had to report the side of the last target, their "left last" responses were recoded as "right first" responses and their "right last" responses were recoded as "left first" responses.

\section{Results}

In the arithmetic task, participants were slower and made more errors when they solved subtraction compared to addition problems-mean $\mathrm{RL} \pm S D=2,585 \pm 53 \mathrm{~ms}$ vs. $2,554 \pm 53$ $\mathrm{ms} ; F(1,10.503)=11.897, p=.001$; mean $\mathrm{ER} \pm S D=6 \% \pm$ $0.8 \%$ vs. $4 \% \pm 0.6 \%, F(1,11.209)=9.586, p=.002$.

In the TOJ task, the results were similar to those obtained in the previous experiments. As illustrated in Figs. 4 and 5, addition elicited more rightward responses than subtraction, regardless of instruction. When the instruction required participants to report the first target, addition elicited more rightward responses than subtraction. When the instruction required

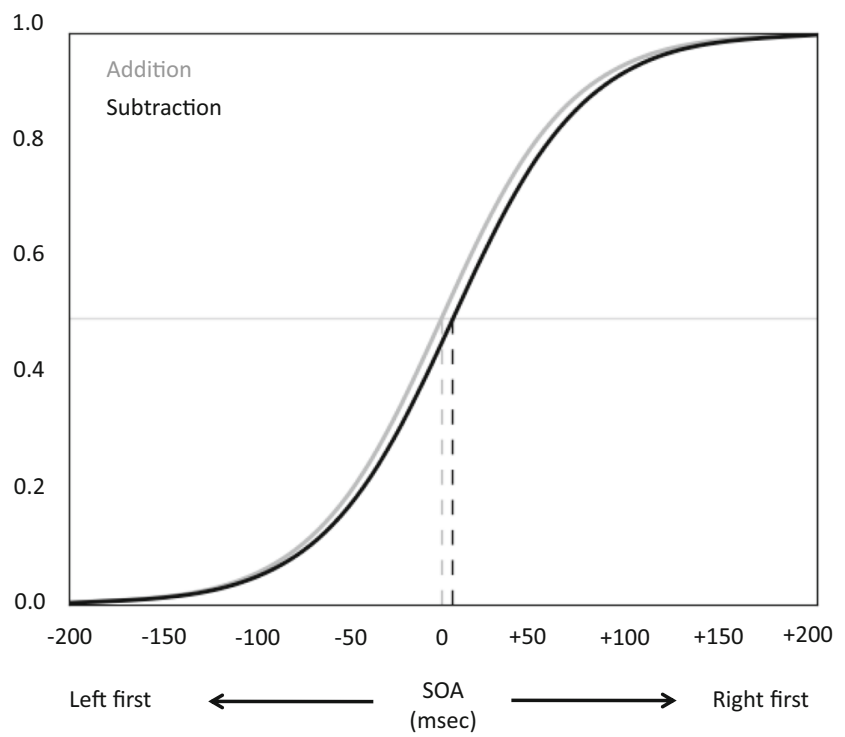

Fig. 4 Probability of reporting the right target as the first, in Experiment 3 , as a function of operation (addition vs. subtraction) and SOA between the left and right targets (from -200 to +200 ). Negative and positive SOAs refer to left-side and right-side precedence, respectively. Dashed lines indicate the PMU for addition (gray) and subtraction (black) participants to report the last target, addition also elicited more rightward responses than subtraction (see Table 3 ). This was reflected in an interaction between OPERATION and INSTRUCTION after all responses were recoded to indicate the probability of reporting the right target as the first, $F(1,10497)$ $=11.454, p=.001$. When participants had to indicate the side of the first target, they reported more often the right target as the first to appear for addition than for subtraction $(p=.015)$. When asked to report which of the target appeared last, participants reported less often the right target as the last for addition than for subtraction $(p=.024)$. The GLMM analysis also showed a significant effect of SOA, $F(1,10497)=$ $1,900.209, p<.001$, indicating that "right target first" responses increased from $0.2 \%$ at SOA $-200 \mathrm{~ms}$ (i.e., left before right) to $99.8 \%$ at $\mathrm{SOA}+200 \mathrm{~ms}$ (i.e., right before left), and an interaction between SOA and INSTRUCTION, $F(1,10497)=$ $10.905, p=.001$, indicating that temporal order was better discriminated when the instruction required participants to focus on the last rather than the first target. There was no interaction between SOA and OPERATION, $F(1,10497)=$ $1.294, p=.255$, and no triple interaction, $F(1,10497)=$

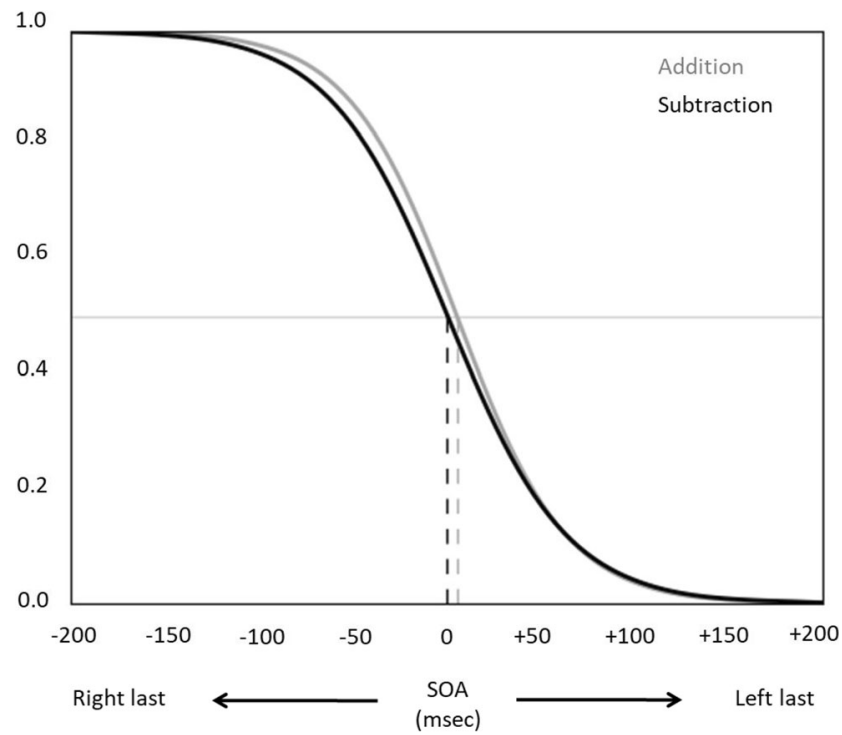

Fig. 5 Probability of reporting the right target as the last, in Experiment 3, as a function of operation (addition vs. subtraction) and SOA between the left and right targets (from -200 to +200 ). Negative and positive SOAs refer to left-side and right-side precedence, respectively. Dashed lines indicate the PMU for addition (gray) and subtraction (black) 
Table 3 Probability of pressing right response key in Experiment 3

\begin{tabular}{|c|c|c|c|c|c|c|c|c|c|c|c|c|c|}
\hline \multirow[t]{2}{*}{ Instruction } & \multirow[t]{2}{*}{ Operation } & \multicolumn{12}{|c|}{$\mathrm{SOA}(\mathrm{ms})$} \\
\hline & & -200 & -100 & -50 & -33 & -17 & 0 & 17 & 33 & 50 & 100 & 200 & Total \\
\hline \multirow[t]{2}{*}{ First } & Addition & $<.01$ & .05 & .20 & .29 & .39 & .50 & .62 & .72 & .81 & .95 & $>.99$ & .50 \\
\hline & Subtraction & $<.01$ & .05 & .17 & .06 & .35 & .46 & .58 & .68 & .78 & .94 & $>.99$ & .48 \\
\hline \multirow[t]{2}{*}{ Second } & Addition & $>.99$ & .97 & .87 & .79 & .69 & .55 & .40 & .28 & .18 & .04 & $<.01$ & .52 \\
\hline & Subtraction & $>.99$ & .96 & .83 & .74 & .64 & .50 & .38 & .27 & .17 & .04 & $<.01$ & .50 \\
\hline
\end{tabular}

$.569, p=.451$. In trials where participants had to report the side of the first target, the PMU was $-1 \mathrm{~ms}$ for addition and $+6 \mathrm{~ms}$ for subtraction. In trials where participants had to report the side of the last target, the PMU was $+6 \mathrm{~ms}$ for addition and $+1 \mathrm{~ms}$ for subtraction.

\section{Discussion}

The results showed that, compared with subtraction, addition biased TOJ toward the "right" response, whether the instruction was to identify the first (Experiment 1) or the last target (Experiment 2) appearing on the screen. If the solving of addition problems involved shifting attention to the right side, it should have increased the probability of reporting "right as first" and "left as last." The results thus undermine the assumption that in standard lateralized detection tasks spatial biases observed immediately after problem presentation reflect attention shifts. They emphasize the need to consider semantic associations linking the addition operator to the right response and the subtraction operator to the left response as the source of spatial biases. These semantic associations seem to exert a pervasive influence on lateralized target detection since the spatial bias initially evidenced with a 200-ms delay between problem offset and target onset (Experiments 1 and 2) was replicated with a 800 -ms delay using a within-subjects design (Experiment 3). While Experiments 1 and 2 suggested that participants might have more resources to focus on the targets after they had solved an addition problem, Experiment 3 showed that the spatial bias was still observable when participants answered the temporal order judgement first and discriminated the targets equally well in the addition and subtraction trials.

Semantic associations occurring along arithmetic processing could not be clearly identified in previous behavioural studies because they were intermingled with spatial attentional shifts. Behavioural studies used to investigate space and arithmetic interactions using horizontally aligned responses that mapped the direction of the expected spatial attention shifts. Generally, participants had to discriminate lateralized targets (e.g., Masson \& Pesenti, 2014), estimate the position of the answer on a left-to-right oriented line (e.g., Pinhas \& Fischer,
2008; Pinheiro-Chagas et al., 2017), or select an answer among probes displayed on a screen (e.g., Knops, Viarouge, \& Dehaene, 2009a; Marghetis et al., 2014). Because attention and response selection were tied in these studies, attributing the origin of the effects to attention shifts or to the semantic congruity between the operation and the response side was not possible. We solved this issue by manipulating the instructions of the TOJ so that the semantic and attention-orienting accounts led to opposite predictions. Semantic associations also account for other results, such as the spatial biases observed during the solving of problems with zero as second operand for which no attention displacement is required (Masson \& Pesenti, 2014; Pinhas \& Fischer, 2008). Our results converge with these data to suggest semantic influences possibly induced by the operator.

How can these results be integrated with previous data linking arithmetic problem solving and attentional processes? Evidence for explicit attention shifts mainly comes from eyetracking studies. Several studies showed that participants looking at a blank screen shift their gaze upward/rightward when counting up and downward/leftward when counting down (Hartmann, Mast, \& Fischer, 2016; see also Loetscher, Bockish, Nicholls, \& Brugger, 2010), and that the gaze moves in opposite directions during the solving of subtraction and addition problems, respectively to the left and to the right (Masson, Letesson, \& Pesenti, 2018). Other studies showed that the manipulation of the locus of attention impacted on arithmetic performance: Inducing reflexive eye movements with optokinetic stimulation has been shown to facilitate the processing of addition problems (Masson, Pesenti, \& Dormal, 2017b). Moreover, flickering distractors presented during the solving of arithmetic problems had an interfering effect on the solving of subtraction and addition problems if they were presented on the left and right side, respectively (Masson \& Pesenti, 2016). Even more compelling, neuropsychological studies of neglect patients showed that the inability to orient attention leftward or rightward impacted respectively on subtraction problem solving (Dormal, Schuller, Nihoul, Pesenti, $\&$ Andres, 2014) and addition problem solving (Masson, Pesenti, Coyette, Andres, \& Dormal, 2017a). These data provide strong evidence that attentional shifts play a functional role in mental arithmetic, but it is worth noting that these shifts 
or their effect are generally identified after the operands and operator have been presented (e.g., $310 \mathrm{~ms}$ after the offset of the problem in Masson et al., 2018), thus when the calculation procedure has already started. It is reasonable to assume that the effect of semantic associations linking the operatorhence the operation - to one side of space takes place before the solving procedure starts. This may orient the choice of the solving procedure (e.g., Liu et al., 2017a, b; Mathieu et al., 2018; Pinhas et al., 2014) and determine the involvement of attention processes in later stages of arithmetic problem solving (e.g., Masson et al., 2018). Deciphering the nature of spatial biases in the early versus late stages of processing will be essential to understand how arithmetic problems are spatially coded before overt attention shifts take place. In lateralized detection tasks, our results suggest that semantic associations are likely to overcome other sources of spatial biases and potentially mask the attention shifts accompanying arithmetic operations even in later stages. In this respect, the present work calls for greater caution in the interpretation of spatial biases in lateralized detection tasks and for the use of more direct measures of attention shifts in future studies.

To conclude, the present results underline the influence of semantic associations in the way we comprehend numbers and arithmetic operations, but they do not evacuate the attention-orienting account. Assuming they may have a heuristic role in cognition (Lakoff \& Nunez, 2000), we propose that semantic associations may contribute to the deployment of the attentional processes involved in the solving procedure. In this view, the activation of the semantic nodes linking a given operation to one side of space could support the deployment of spatial attention toward the location where the answer is represented (Mathieu et al., 2018). This hypothesis requires further investigation, but it grounds the explanation of spacearithmetic interactions in an information-processing theoretic perspective where the fixed associations in semantic memory and the dynamic attention mechanisms triggered by arithmetic problems both contribute to reduce uncertainty about the answer. The solving of arithmetic problems would be analogous to the construction of online probability models incorporating different sources of information, such as the memory of an association between each operator and each side of space, with the aim to progressively narrow down the range of plausible solutions (Zénon, Solopchuk, \& Pezzulo, 2019).

\footnotetext{
Acknowledgements The authors have no conflict of interest to declare. M.A. is a research associate, M.P a senior research associate, at the Fonds National de la Recherche Scientifique (FRS-FNRS, Belgium). N.M. is a postdoctoral researcher funded by grant PDR-FNRS T.0047.18 to MP, and S.S. is a doctoral student funded by grant PDR-FNRS T.0245.16 to MA from the Fonds National de la Recherche Scientifique (FRS-FNRS, Belgium). We thank Pascaline Le Maire, Marion Deldicque, and Stuart Dale for their help in data collection.
}

Data sharing statement The dataset of each experiment is available in the Open Science Foundation repository with a CC-O licence, osf.io/ ef9ky. None of the experiments were preregistered.

\section{References}

Bächtold, D., Baumüller, M., \& Brugger, P. (1998). Stimulus-response compatibility in representational space. Neuropsychologia, 36(8), 731-735. https://doi.org/10.1016/S0028-3932(98)00002-5

Casarotti, M., Michielin, M., Zorzi, M., \& Umiltà, C. (2007). Temporal order judgment reveals how number magnitude affects visuospatial attention. Cognition, 102, 101-117. https://doi.org/10.1016/j. cognition.2006.09.001

Dehaene, S. (1992). Varieties of numerical abilities. Cognition, 4, 1-42. https://doi.org/10.1016/0010-0277(92)90049-N

Dormal, V., Schuller, A. M., Nihoul, J., Pesenti, M., \& Andres, M. (2014). Causal role of spatial attention in arithmetic problem solving: Evidence from left unilateral neglect. Neuropsychologia, 60, 1-9. https://doi.org/10.1016/j.neuropsychologia.2014.05.007

Fias, W., Brysbaert, M., Geypens, F., \& d'Ydewalle, G. (1996). The importance of magnitude information in numerical processing: Evidence from the SNARC effect. Mathematical Cognition, 2(1), 95-110.

Fischer, M. H., Castel, A. D., Dodd, M. D., \& Pratt, J. (2003). Perceiving numbers causes spatial shifts of attention. Nature Neuroscience, 6(6), 555-556. https://doi.org/10.1038/nn1066

Gevers, W., Lammertyn, J., Notebaert, W., Verguts, T., \& Fias, W. (2006). Automatic response activation of implicit spatial information: Evidence from SNARC effect. Acta Psychologica, 122, 221-233. https://doi.org/10.1016/j.actpsy.2005.11.004

Gevers, W., Santens, S., Dhooge, E., Chen, Q., Van den Bossche, L., Fias, W., \& Verguts, T. (2010). Verbal-spatial and visuospatial coding of number-space interactions. Journal of Experimental Psychology: General, 139(1), 180-190. https://doi.org/10.1037/a0017688

Hartmann, M., Mast, F. W., \& Fischer, M. H. (2015). Spatial biases during mental arithmetic: Evidence from eye movements on a blank screen. Frontiers in Psychology, 6, 12. https://doi.org/10.3389/ fpsyg.2015.00012

Hartmann, M., Mast, F. W., \& Fischer, M. H. (2016). Counting is a spatial process: Evidence from eye movements. Psychological Research, 80(3), 399-409. https://doi.org/10.1007/s00426-015-0722-5

Hayes, J. R. (1973). On the function of visual imagery in elementary mathematics. In W. G. Chase (Ed.), Visual information processing (pp. 177-214). New York, NY: Academic Press. https://doi.org/10. 1016/B978-0-12-170150-5.50010-X

Hubbard, E. M., Piazza, M., Pinel, P., \& Dehaene, S. (2005). Interactions between number and space in parietal cortex. Nature Reviews Neuroscience, 6(6), 435-448. https://doi.org/10.1038/nrn1684

Knops, A., Thirion, B., Hubbard, E. M., Michel, V., \& Dehaene, S. (2009b). Recruitment of an area involved in eye movements during mental arithmetic. Science, 324, 1583-1585. https://doi.org/10. 1126/science. 1171599

Knops, A., Viarouge, A., \& Dehaene, S. (2009a). Dynamic representations underlying symbolic and nonsymbolic calculation: Evidence from the operational momentum effect. Attention, Perception, \& Psychophysics, 71, 803-821. https://doi.org/10.3758/APP.71.4.803

Lakoff, G., \& Nunez, R. (2000). Where mathematics comes from: How the embodied mind brings mathematics into being. New York, NY: Basic Books.

Liu, D., Cai, D., Verguts, T., \& Chen, Q. (2017b). The time course of spatial attention shifts in elementary arithmetic. Scientific Reports, 7(1), 921. https://doi.org/10.1038/s41598-017-01037-3

Liu, D., Verguts, T., Li, M., Ling, Z., \& Chen, Q. (2017a). Dissociated spatial-arithmetic associations in horizontal and vertical 
dimensions. Frontiers in Psychology. https://doi.org/10.3389/fpsyg. 2017.01741

Loetscher, T., Bockisch, C. J., Nicholls, M. E., \& Brugger, P. (2010). Eye position predicts what number you have in mind. Current Biology, 20(6), R264-R265. https://doi.org/10.1016/j.cub.2010.01.015

Marghetis, T., Núñez, R., \& Bergen, B. K. (2014). Doing arithmetic by hand: Hand movements during exact arithmetic reveal systematic, dynamic spatial processing. The Quarterly Journal of Experimental Psychology, 67(8), 1579-1596. https://doi.org/10.1080/17470218. 2014.897359

Masson, N., Letesson, C., \& Pesenti, M. (2018). Time course of overt attentional shifts in mental arithmetic: Evidence from gaze metrics. The Quarterly Journal of Experimental Psychology, 71(4), 10091019. https://doi.org/10.1080/17470218.2017.1318931

Masson, N., \& Pesenti, M. (2014). Attentional bias induced by solving simple and complex addition and subtraction problems. The Quarterly Journal of Experimental Psychology, 67, 1514-1526. https://doi.org/10.1080/17470218.2014.903985

Masson, N., \& Pesenti, M. (2016). Interference of lateralized distractors on arithmetic problem solving: A functional role for attention shifts in mental calculation. Psychological Research, 80, 640-651. https:// doi.org/10.1007/s00426-015-0668-7

Masson, N., Pesenti, M., Coyette, F., Andres, M., \& Dormal, V. (2017a). Shifts of spatial attention underlie numerical comparison and mental arithmetic: Evidence from a patient with right unilateral neglect. Neuropsychology, 31(7), 822-833. https://doi.org/10.1037/ neu0000361

Masson, N., Pesenti, M., \& Dormal, V. (2017b). Impact of optokinetic stimulation on mental arithmetic. Psychological Research, 81(4), 840-849. https://doi.org/10.1007/s00426-016-0784-z

Mathieu, R., Epinat-Duclos, J., Sigovan, M., Breton, A., Cheylus, A., Fayol, M., ... Prado, J. (2018). What's behind a "+" sign? Perceiving an arithmetic operator recruits brain circuits for spatial orienting. Cerebral Cortex, 28(5), 1673-1684. https://doi.org/10. 1093/cercor/bhx064

Mathieu, R., Gourjon, A., Couderc, A., Thevenot, C., \& Prado, J. (2016). Running the number line: Rapid shifts of attention in single-digit arithmetic. Cognition, 146, 229-239. https://doi.org/10.1016/j. cognition.2015.10.002

McCrink, K., Dehaene, S., \& Dehaene-Lambertz, G. (2007). Moving along the number line: Operational momentum in nonsymbolic arithmetic. Perception \& Psychophysics, 69, 1324-1333. https://doi. org/10.3758/BF03192949
Peirce, J. W. (2007). PsychoPy-Psychophysics software in Python. Journal of Neuroscience Methods, 162(1/2), 8-13. https://doi.org/ 10.1016/j.jneumeth.2006.11.017

Pinhas, M., \& Fischer, M. (2008). Mental movements without magnitude? A study of spatial biases in symbolic arithmetic. Cognition, 109(3), 408-415. https://doi.org/10.1016/j.cognition.2008.09.003

Pinhas, M., Shaki, S., \& Fischer, M.H., (2014). Heed the signs: Operation signs have spatial associations. The Quarterly Journal of Experimental Psychology, 67(8), 1527-1540. https://doi.org/10. 1080/17470218.2014.892516

Pinheiro-Chagas, P., Dotan, D., Piazza, M., \& Dehaene, S. (2017). Finger tracking reveals the covert stages of mental arithmetic. Open Mind, l(1), 30-41. https://doi.org/10.1162/OPMI a 00003

Proctor, R. W., \& Cho, Y. S. (2006). Polarity correspondence: A general principle for performance of speeded binary classification tasks. Psychological Bulletin, 132(3), 416-442. https://doi.org/10.1037/ 0033-2909.132.3.416

Stelmach, L. B., \& Herdman, C. M. (1991). Directed attention and perception of temporal order. Journal of Experimental Psychology: Human Perception and Performance, 17, 539-550. https://doi.org/ 10.1037/0096-1523.17.2.539

van Dijck, J. P., \& Fias, W. (2011). A working memory account for spatial-numerical associations. Cognition, 119(1), 114-119. https://doi.org/10.1016/j.cognition.2010.12.013

Wiemers, M., Bekkering, H., \& Lindemann, O. (2014). Spatial interferences in mental arithmetic: Evidence from the motion-arithmetic compatibility effect. The Quarterly Journal of Experimental Psychology, 67(8), 1557-1570. https://doi.org/10.1080/17470218. 2014.889180

Zago, L., Pesenti, M., Mellet, E., Crivello, F., Mazoyer, B., \& TzourioMazoyer, N. (2001). Neural correlates of simple and complex mental calculation. NeuroImage, 13(2), 314-327. https://doi.org/10. 1006/nimg.2000.0697

Zago, L., Petit, L., Turbelin, M. R., Andersson, F., Vigneau, M., \& Tzourio-Mazoyer, N. (2008). How verbal and spatial manipulation networks contribute to calculation: An fMRI study. Neuropsychologia, 46(9), 2403-2414. https://doi.org/10.1016/j. neuropsychologia.2008.03.001

Zénon, A., Solopchuk, O., \& Pezzulo G. (2019). An informationtheoretic perspective on the costs of cognition. Neuropsychologia, 123, 5-18. https://doi.org/10.1016/j.neuropsychologia.2018.09.013

Publisher's note Springer Nature remains neutral with regard to jurisdictional claims in published maps and institutional affiliations. 